\title{
Evaluation of Procalcitonin, CRP and Blood Culture in the Diagnosis of Neonatal Sepsis
}

\author{
Rashmi Padmanabha Mahale, Morubagal Raghavendra Rao* and \\ Sowmya Govindanahalli Shivappa
}

\section{Department of Microbiology, JSS Medical College, Jss academy of Higher Education and Research, Shivarathreswara Nagar Mysore-570015, Karnataka, India}

*Corresponding author

\begin{tabular}{|l|}
\hline Key w o r d s \\
Blood culture, \\
Neonatal sepsis, C- \\
reactive protein, \\
Procalcitonin
\end{tabular}

\section{Introduction}

Neonatal sepsis is invasive infection, usually bacterial, occurring during the neonatal period. Sepsis is a major cause of morbidity and mortality among neonates, especially in the developing countries (Shah et al., 2006). An overall incidence of the culture proven sepsis of 1-8 cases per 1000 live births from the developed countries and 4.8-20.7 per 1000 
live births from India has been reported (Ramesh Bhat and Lincy, 2011).

Neonatal sepsis may be categorized as earlyonset or late-onset. Early onset occurs within first 5-7 days postnatally and is due to infection occurring through transplacental transmission within the uterus or an ascending infection from the cervix of the mother by organisms that colonize the genito-urinary tract. The neonate acquires the microorganisms as it passes through the colonized birth canal at delivery. Of newborns with early-onset sepsis, $85 \%$ present within 24 hours, $5 \%$ present at 24-48 hours, and a smaller percentage present within 48-72 hours. Onset is most rapid in premature neonates.

The microorganisms most commonly associated with early-onset infection include Group B Streptococcus, Escherichia coli, Coagulase-negative Staphylococcus, Haemophilus influenza and Listeria monocytogenes (Klinger et al., 2009). The case fatality rate in early onset neonatal sepsis ranges from $16.7 \%$ to $19.4 \%$ (Baltimore et al., 2001). Late onset sepsis develops 10-30 days after birth and the source of infection is the care-giving environment. Organisms that have been implicated in causing late-onset sepsis include Coagulase-negative staphylococcus, Staphylococcus aureus, E. coli, Klebsiella, Pseudomonas, Enterobacter, Candida, Group B Streptococcus, Serratia, Acinetobacter and anaerobic bacteria (Van den Hoogen et al., 2010).

Most common predisposing and precipitating factors are premature rupture of membrane (PROM), prolonged second stage of labour or other maternal causes such as maternal fever within 2 weeks prior to delivery, meconium stained amniotic fluid (MSAF), foul smelling liquor and instrumental delivery. The foetal factors include low birth weight, prematurity and Apgar score (Shah et al., 2006).
Clinical manifestations of neonatal sepsis are non-specific and include lethargy, poor feeding, temperature instabilities, respiratory distress, apnoea and shock (Lisa Ross De Camp et al., 2009). However, these clinical signs also indicate the presence of many nonseptic conditions like hypoglycaemia, hypothermia, electrolyte imbalance, inborn errors of metabolism, etc. Also, some neonates, especially premature ones may have very subtle features or even no features. Therefore, clinical diagnosis of sepsis is difficult and laboratory help is required.

In the laboratory, blood culture, owing to its specificity is considered the gold standard for detection of sepsis. However, it is very time consuming and there is always a possibility of false positivity, due to sample contamination or false negativity, when the neonate has already been exposed to antibiotic agents during labour. Also, studies on sepsis have underlined the concern about possible culture negative clinical sepsis, particularly in the setting of increasing maternal antibiotic use (Stoll et al., 1996).

Recent studies have showed promising results with estimation of C-Reactive Protein and Procalcitonin. C-reactive protein (CRP) is an acute-phase reactant that is synthesized by the liver within six hours after the onset of inflammation and tissue necrosis. Its physiological role is to bind to phosphocholine expressed on the surface of dead or dying cells and some types of bacteria in order to activate the complement system via the C1Q complex (Thompson et al., 1999). Its rapid synthesis, short half-life and rapid decline with recovery, together with an association between increased blood values and serious bacterial infections, have made the CRP test popular. This test is often requested to help discriminate viral infections from bacterial infections or monitor the response to antibiotics (Joan M. Hengst, 2012). 
It has been recently reported that procalcitonin (PCT), the prohormone of calcitonin, increases markedly in septic conditions and it appears to be a good predictor of infection severity. In this case, Procalcitonin is secreted mainly by the neuroendocrine cells of the lungs and intestine in response to bacterial endotoxin or inflammatory cytokines (TNF, IL-6) (Maruna et al., 2000).

It does not rise significantly with viral or noninfectious inflammations, hence it is highly specific to bacterial sepsis. Furthermore, PCT is released into the circulation within 3 hours of onset of infection, plateaus at 6 hours, and remains elevated for 24 hours. This makes PCT a promising new agent for early and sensitive identification of infected neonates (José B López Sastre et al., 2007).

In view of the above diagnostic merits of CRP and PCT, this study aims at investigating the feasibility of adapting these biomarkers into routine tests for neonatal sepsis along with the standard blood culture in the hospital settings. Lastly, an antibiotic sensitivity pattern needs to be drawn, to avoid re-emergence of infection and development of drug resistant strains.

\section{Materials and Methods}

$3 \mathrm{ml}$ of venous blood was collected employing strict aseptic precautions from all babies admitted to the NICU in our hospital during the study period. $2 \mathrm{ml}$ of blood was aseptically added to BacT/ ALERT PF culture bottle. The bottle was loaded into the BacT/ ALERT Microbial Detection System after entering the relevant patient data. Blood culture bottle flagging indicates bacterial growth.

Sub cultures were made from such bottles on to MacConkey and Blood agar plates. Inoculated plates were incubated at 37 degrees for $24 \mathrm{hrs}$. Colony formed on these culture media were identified by using the standard protocol. The antibiotic susceptibility for the isolates was tested by Kirby Bauer method and CLSI guidelines were followed during testing and interpretation of susceptibility or resistance to the antibiotics.

Remaining blood sample was added to plain vacutainer (BD) and allowed to clot. The serum separated was used for PCT estimation by immunochromatography assay and CRP estimation by chemiluminescence method. Manufacturer's instructions were followed during testing and interpretation of the two serological acute phase markers.

\section{Results and Discussion}

In our study 50 blood samples were collected from neonates admitted to the NICU. Out of these, $15(30 \%)$ were preterm and the remaining $35(70 \%)$ were term babies. All the subjects in our study were evaluated for early onset sepsis that is, sepsis occurring before 7 days after delivery. Graph 1 depicts the number of cases evaluated on day 1 to day 7 after birth of the baby:

Various risk factors that were present in our study population are shown in table 1.

Most common risk factor of suspected septicaemia in our study was birth asphyxia (32\%), followed by preterm birth $(28 \%)$ and Meconium Stained Amniotic Fluid (28\%). 3 deaths were reported during the study period in the study group.

Table 2 shows various concentrations of PCT in the 50 samples that were tested.

$16(32 \%)$ sample from clinically suspected sepsis cases, PCT was negative and from all these cases blood culture also yielded no growth. PCT was positive in all blood culture positive (proven sepsis) cases. 
Table.1 Various risk factors in study population

\begin{tabular}{|c|c|c|c|}
\hline SI. No. & Risk Factors & Number of subjects & Percentage \\
\hline 1. & Meconium Stained Amniotic Fluid alone & 10 & $20 \%$ \\
\hline 2. & Birth Asphyxia alone & 11 & $22 \%$ \\
\hline 3. & Preterm birth alone & 9 & $18 \%$ \\
\hline 4. & PROM alone & 2 & $4 \%$ \\
\hline 5. & Obstructed labor alone & 1 & $2 \%$ \\
\hline 6. & Hyperbilirubinaemia alone & 3 & $6 \%$ \\
\hline 7. & Congenital anomalies alone & 2 & $4 \%$ \\
\hline 8. & Fever & 1 & $2 \%$ \\
\hline 9. & Preterm birth with obstructed labor & 1 & $2 \%$ \\
\hline 10. & Neonatal depression with MSAF & 1 & $2 \%$ \\
\hline 11. & Preterm birth with neonatal depression & 2 & $4 \%$ \\
\hline 12. & Neonatal depression with birth asphyxia & 2 & $4 \%$ \\
\hline 13. & Preterm birth with MSAF & 2 & $4 \%$ \\
\hline 14. & Birth asphyxia with PROM & 1 & $2 \%$ \\
\hline 15. & Birth asphyxia with MSAF & 2 & $4 \%$ \\
\hline
\end{tabular}

Table.2 PCT concentration of serum samples

\begin{tabular}{|c|c|c|c|}
\hline S. No. & PCT Concentration $(\mu \mathrm{gg} / \mathrm{L})$ & Number of subjects & Percentage \\
\hline 1. & 0 & 16 & $32 \%$ \\
\hline 2. & $0-0.5$ & 03 & $6 \%$ \\
\hline 3. & $\geq 0.5-<2$ & 13 & $26 \%$ \\
\hline 4. & $\geq 2-<10$ & 05 & $10 \%$ \\
\hline 5. & $\geq 10$ & 13 & $26 \%$ \\
\hline
\end{tabular}

Table.3 CRP concentration of serum samples

\begin{tabular}{|l|l|l|l|l|l|l|l|l|l|l|l|l|}
\hline SI. No. & 1 & 2 & 3 & 4 & 5 & 6 & 7 & 8 & 9 & 10 & 11 & 12 \\
\hline $\begin{array}{l}\text { CRP } \\
(\mathrm{mg} / \mathrm{dL})\end{array}$ & 16.15 & 41.8 & 37.0 & 31.2 & 48 & 91.45 & 62.36 & 24.7 & 120 & 62.3 & 62.36 & 41.8 \\
\hline
\end{tabular}

Table.4 For Gram positive organism's sensitivity pattern

\begin{tabular}{|l|l|l|l|l|l|l|l|}
\hline $\begin{array}{c}\text { Case } \\
\text { No. }\end{array}$ & $\begin{array}{c}\text { Causative } \\
\text { agent }\end{array}$ & Penicillin & Gentamicin & Rifampicin & Ciprofloxacin & Linazolid & Cefoxitin \\
\hline $\mathbf{1}$ & S. aureus & R & S & R & S & S & R \\
\hline $\mathbf{2}$ & S. aureus & R & R & R & S & S & S \\
\hline 3 & S. aureus & S & S & S & S & S & S \\
\hline 4 & S. aureus & R & S & S & S & S & R \\
\hline
\end{tabular}


Table.5 For Gram negative organism's sensitivity pattern

\begin{tabular}{|c|c|c|c|c|c|c|c|c|}
$\begin{array}{c}\text { Case } \\
\text { No. }\end{array}$ & $\begin{array}{c}\text { Causative } \\
\text { agent }\end{array}$ & \multicolumn{8}{|c|}{$\begin{array}{c}\text { Antibiotic Sensitivity Pattern } \\
\text { Ampicillin Amikacin }\end{array}$} \\
\hline $\mathbf{1}$ & E. coli & $\mathrm{R}$ & $\mathrm{S}$ & $\mathrm{S}$ & $\mathrm{S}$ & $\mathrm{S}$ & $\mathrm{R}$ & $\mathrm{R}$ \\
\hline $\mathbf{2}$ & E. coli & $\mathrm{R}$ & $\mathrm{S}$ & $\mathrm{S}$ & $\mathrm{S}$ & $\mathrm{R}$ & $\mathrm{R}$ & $\mathrm{R}$ \\
\hline $\mathbf{3}$ & E. coli & $\mathrm{R}$ & $\mathrm{S}$ & $\mathrm{S}$ & $\mathrm{S}$ & $\mathrm{S}$ & $\mathrm{R}$ & $\mathrm{R}$ \\
\hline $\mathbf{4}$ & K.pneumoniae & $\mathrm{R}$ & $\mathrm{S}$ & $\mathrm{S}$ & $\mathrm{S}$ & $\mathrm{S}$ & $\mathrm{S}$ & $\mathrm{S}$ \\
\hline $\mathbf{5}$ & K.pneumoniae & $\mathrm{R}$ & $\mathrm{R}$ & $\mathrm{R}$ & $\mathrm{S}$ & $\mathrm{S}$ & $\mathrm{S}$ & $\mathrm{S}$ \\
\hline $\mathbf{6}$ & Pseudomonas & $\mathrm{S}$ & $\mathrm{S}$ & $\mathrm{R}$ & $\mathrm{S}$ & $\mathrm{R}$ & $\mathrm{R}$ & $\mathrm{R}$ \\
\hline $\mathbf{7}$ & Acinetobacter & $\mathrm{R}$ & $\mathrm{R}$ & $\mathrm{S}$ & $\mathrm{S}$ & $\mathrm{S}$ & $\mathrm{S}$ & $\mathrm{S}$ \\
\hline $\mathbf{8}$ & Citrobacter & $\mathrm{R}$ & $\mathrm{S}$ & $\mathrm{S}$ & $\mathrm{S}$ & $\mathrm{S}$ & $\mathrm{S}$ & $\mathrm{S}$ \\
\hline
\end{tabular}

Graph.1 Showing day wise evaluated of cases

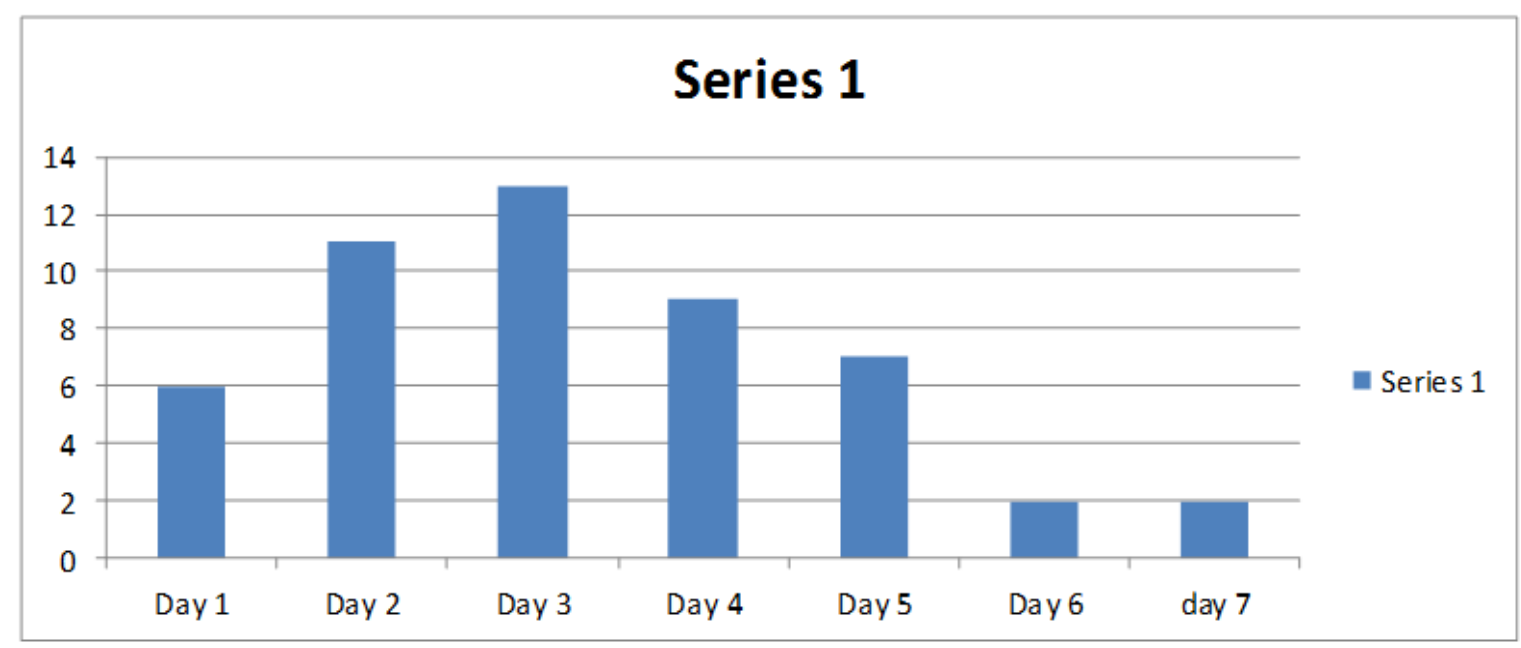

Graph.2 Showing different types of bacteria isolated from blood culture

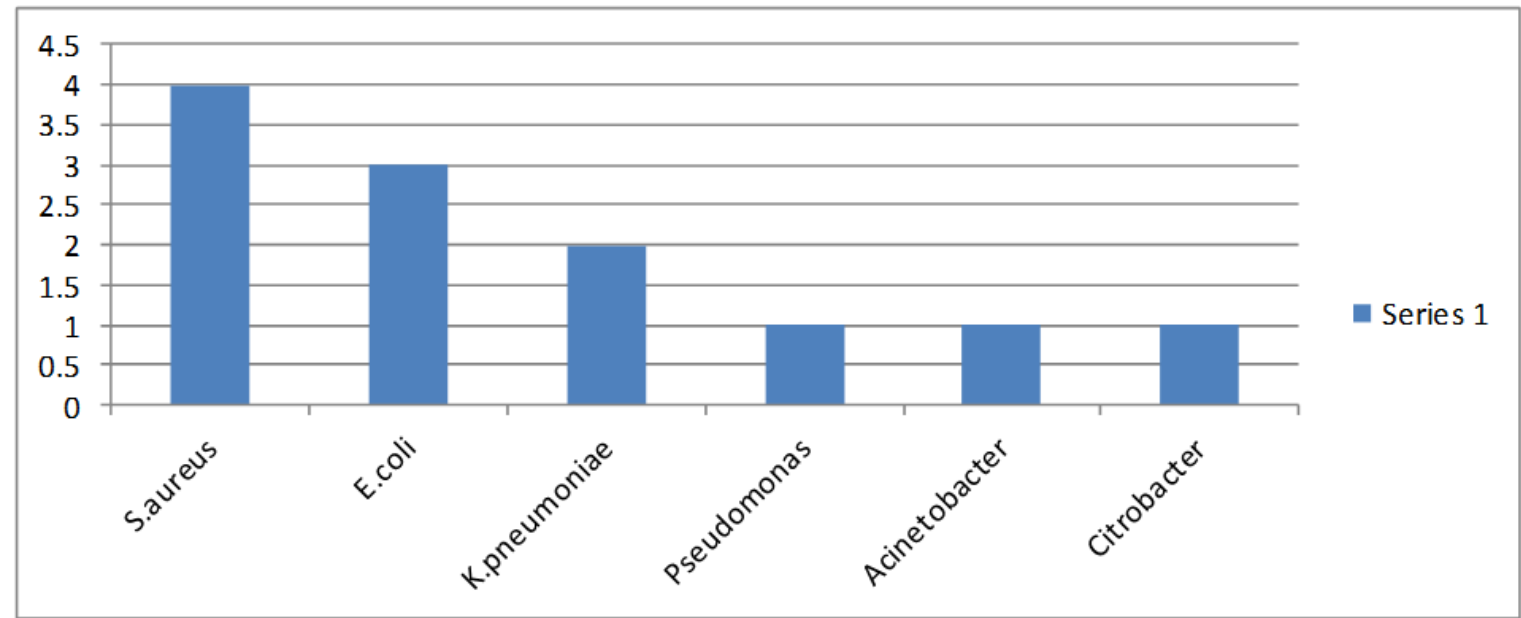


Out of 50 samples tested for CRP, 33(66\%) were positive. A wide range of CRP values were obtained, ranging from $1.83 \mathrm{mg} / \mathrm{dL}$ to $120 \mathrm{mg} / \mathrm{dL}$. Out of the 34 PCT positive cases, $33(97.05 \%)$ were also CRP positive.

CRP was positive with high values in all the proven sepsis cases. However, 21(63.63\%) cases which were blood culture negative gave a positive CRP. Table 3 shows the different CRP concentrations in the blood culture positive cases.

Blood culture was positive in $12(24 \%)$ cases. Graph 2 shows the various causative agents that were detected by culture in our study

The most common etiological agent was Staphylococcus aureus.

Table 4 and 5 shows the antibiotic sensitivity pattern of Gram positive and Gram negative bacteria isolated. Out of $4 S$. aureus isolates 2 were MRSA. Among E. coli all the 3 isolates were resistant to Cefotaxime and sensitive to Imipenum.

Neonatal sepsis remains a diagnostic and treatment challenge for modern neonatal care providers, especially in developing countries where highest incidence and the highest mortality rates are reported. Infants presenting with clinical signs of infection are evaluated with a variety of diagnostic tests and treatment with broad-spectrum antibiotics initiated until a definitive diagnosis can be made. Isolation of microorganism(s) from one or more blood cultures is the gold standard to establish a definitive diagnosis of neonatal sepsis (Pourcyrous et al., 1993).

The sole use of blood culture to diagnose neonatal infection has a number of limitations. It may take 24 to 72 hours to obtain culture results (Garcia-Prats et al., 2000). The sensitivity of blood cultures may be impaired by exposure to intrapartum antibiotics, which are administered to $15 \%$ to $40 \%$ of mothers in labor (Bromberger et al., 2000). Intrapartum antibiotic exposure can result in a partially treated infant, delaying the onset of clinical signs and symptoms of infection and further complicating the expedient definitive diagnosis of early-onset sepsis in the infant. With the development of multiple drug-resistant bacteria and the cost of therapy with multiple antibiotics, the ability to diagnose or rule out sepsis is an essential tool to limit inappropriate antibiotic exposure (Philip and Mills, 2001).

Variety of laboratory tests, other than blood culture has been developed to enhance the early and accurate identification and treatment of infants with suspected sepsis. In present study blood culture and two biomarkers CRP and PCT were evaluated.

Out of the 50 clinically suspected cases of neonatal sepsis, 12 were culture positive which accounts to $24 \%$ of cases in our study. This is comparable to a study conducted by (Kapoor et al., 2005) and (Dechen C. Tsering et al., 2011) who reported $20 \%$ and $22 \%$ respectively of culture proven cases of neonatal sepsis. A study conducted by (Kurien Ani Kuruvi et al., 1998)' in Vellore reported a mortality rate of $16.7 \%$ and $13.6 \%$ in early and late onset sepsis respectively, unlike in our study where there were only $3(6 \%)$ deaths related to neonatal sepsis. Adequate anticipation, early diagnosis and treatment with appropriate antibiotics have contributed to this low level of mortality.

In gram negative bacteria, $E$. coli was the most common isolate (25\%) followed by Klebsiella (16.66\%), Psuedomonas (8.33\%), Acinetobacter (8.33\%) and Citrobacter (8.33\%). Staphylococcus aureus (33.33\%) was responsible for sepsis among gram positive organisms. This was comparable to 
the study conducted by (Dechen C. Tsering et al., 2011), however in their study Enterobactor species was leading cause of sepsis among gram negative bacteria. The antimicrobial sensitivity pattern differs in different studies as well as at different times in the same hospital in India and in overseas studies (Dechen C. Tsering et al., 2011) which results from indiscriminate use of antibiotics. In present study all the $S$. aureus isolates were sensitive to Ciprofloxacin and Linazolid and only $25 \%$ were sensitive to Penicillin. This high rate of resistance to Penicillin was also seen in a study conducted in Lucknow (Roy et al., 2002) All gram negative bacteria were sensitive to Imipenum, $75 \%$ to Amikacin and Ciproflaxacin.

Among the various risk factors that predispose the neonates to sepsis, the most important risk factor in our study was birth asphyxia (32\%), followed by preterm birth (28\%) and meconium stained amniotic fluid (28\%) which is comparable to a study in Sikkim (Dechen C. Tsering et al., 2011) while (Kurien Ani Kuruvi et al., 1998) reported meconium stained liquor as the most common risk factor.

PCT was positive in $34(68 \%)$ out of 50 cases. PCT showed a false positive rate of $64.7 \%$, that is PCT was positive in spite of blood culture being negative, but the values of PCT in these cases ranged between $0.5-<10 \mu \mathrm{g} / \mathrm{L}$. 6 cases showed significant levels in spite of being culture negative (significant levels being taken as $>2-\geq 10 \mu \mathrm{g} / \mathrm{L}$. 66\% (33) cases were positive for CRP in our study. $55.26 \%$ (21) cases which were blood culture negative were positive for CRP. Only one case which was blood culture negative with CRP negative gave a false positive PCT, however the PCT level was between $0.5-2 \mu \mathrm{g} / \mathrm{L}$.

False positive CRP results were seen in $55.26 \%$ of cases and false positive PCT test was seen in $64.7 \%$ of cases. This false positive raise in PCT levels may be attributed to the physiological peak among uninfected neonates (José B López Sastre et al., 2007). In the present study we have not found any advantage of PCT over CRP which is comparable with the conclusions of many authors (Biommendahl et al., 2002; Naglaa F. Boraey et al., 2012). We would like to conclude that blood culture even though considered as gold standard it is time consuming and at times gives false negative results due to administration of antibiotics. PCT and CRP are reliable markers which aid in diagnosis of neonatal sepsis, which have the same diagnostic accuracy. CRP, when compared to PCT is affordable and can be conveniently used as a marker for the diagnosis of neonatal sepsis, especially in developing countries with poor resources.

\section{References}

Baltimore RS, Huie SM, Meek JI, Schuchat A, O'Brein KL. Early-onset of neonatal sepsis in the era of group B Streptococcal prevention. Paediatrics 2001; 108: 1094-98.

Biommendahl J, Janas M, Laina S, Miettinen A, Ashorn P: Comparison of PCT with CRP and differential WBC Count for diagnosis of culture proven neonatal sepsis. Scand J infection dis 2002, 34: 620-622

Bromberger P, Lawrence J, Braun D, Saunders B, Contreras R, et al., The influence of intrapartum antibiotics on the clinical spectrum of early-onset group B streptococcal infection in term infants. Pediatrics. 2000; 106: 244-250.

Dechen C Tsering, L Chanchal, Ranabir Pal, Sumit Kar. Bacteriological Profile of Septicaemia and the Risk Factors in neonates and infants in Sikkim. Journal of Global Infectious Diseases 2011; Volume 3; Issue 1: 42-45 
Garcia-Prats J, Cooper R, Schneider V, Stager C, Hansen T. Rapid detection of microorganisms in blood cultures of newborn infants utilizing an automated blood culture system. Pediatrics. 2000; 105: 523-527.

Joan M. Hengst, RNC, MSN, ARNP- The Role of C-Reactive Protein in the Evaluation and Management of Infants With Suspected Sepsis (2012) Nabulsi et al., BMC Pediatrics 12:140

José B López Sastre, David Pérez Solís, Vicente Roqués Serradilla, Belén Fernández Colomer, Gil D Coto Cotallo and Grupo de Hospitales CastrilloEvaluation of procalcitonin for diagnosis of neonatal sepsis of vertical transmission- Published: 26 February 2007 BMC Pediatrics, 7:9 doi:10.1186/1471-2431-7-9

Kapoor L, Randhava VS, Deb M. Microbiological profile of Neonatal Septicaemia in a Paediatric Hospital in Delhi. Journal of Communicable diseases Sept 2005; 37(3):227-232

Klinger G, Levy I, Sirota L, et al., Epidemiology and risk factors for early onset sepsis among very-lowbirthweight infants. Am $J$ Obstet Gynecol. 2009 Jul. 201(1): 38.e1-6. (Medline).

Kurien Ani Kuruvi A, Swati Pi ai, Mary Jesudason and Atanu Kumar Jana. Bacterial profile of sepsis in a Neonatal Unit in South India. Indian Paediatrics 1998; 35:851-858

Lisa Ross De Camp, Amanda F. Dempsey, Beth A. Tarini- Neonatal Sepsis: Looking Beyond the Blood CultureEvaluation of a Study of Universal Primer Polymerase Chain Reaction for Identification of Neonatal Sepsis; Published: Arch Pediatr Adolesc Med. 2009; 163(1): 12-14. doi: 10.1001/archpediatrics. 2008. 515.
Maruna et al., - Physiology and Genetics of Procalcitonin- Published: Physiology Research 49 (Suppl. 1): S57-S61, 2000.

Naglaa F. Boraey, Abeer Sheneef, Mohammad Abd-Allah Mohammad, Laila M. Yousef. Procalcitonin and CReactive Protein as Diagnostic Markers of Neonatal Sepsis. Australian Journal of Basic and Applied Sciences, 6(4): 108-114, 2012

Philip A, and Mills P. Use of C-reactive protein in minimizing antibiotic exposure: experience with infants initially admitted to a well-baby nursery. Pediatrics. 2000. Available at http://www.pediatrics.org/cgi/content/fu 11/106/1/e4. Accessed January 16, 2001.

Pourcyrous M, Bada H, Korones S, Baselski V, Wong S. Significance of serial Creactive protein responses in neonatal infection and other disorders. Pediatrics. 1993; 92: 431-435.

Ramesh Bhat Y, and Lincy P Baby-Early Onset of Neonatal Sepsis: Analysis of the Risk Factors and the Bacterial Isolates by Using the BacT Alert System (2011) Journal of Clinical and Diagnostic Research. 2011 November (Suppl-2), Vol-5(7): 1385-1388

Roy I, Jain A, Kumar M, Agarwal SK. Bacteriology of Neonatal Septicaemia in a Tertiary Care Hospital of Northern India. Indian J Med Microbiol 2002; 20: 156-159

Shah GS, Budhathoki S, Das BK, Mandal RN- Risk Factors in early neonatal sepsis (2006), Kathmandu University Medical Journal, Vol. 4, No. 2, Issue 14, 187-191

Shah GS, Budhathoki S, Das BK, Mandal RN- Risk Factors in early neonatal sepsis (2006), Kathmandu University Medical Journal, Vol. 4, No. 2, Issue 14, 187-191

Stoll BJ, Gorgon T, Korones SB, et al., Early onset sepsis in very low birth 
weight neonates: A report from the National Institute of Child Health and Human Development; Neonatal research network. J Paediatric 1996; 129:72-88

Thompson D, Pepys MB, Wood SP (Feb 1999). "The physiological structure of human C-reactive protein and its complex with phosphocholine".
Structure. 7 (2): 169-77. doi:10.1016/S0969-2126(99)80023-9.

Van den Hoogen A, Gerards LJ, VerboonMaciolek MA, Fleer A, Krediet TG. Long-term trends in the epidemiology of neonatal sepsis and antibiotic susceptibility of causative agents. Neonatology. 2010. 97(1): 22-8. (Medline).

\section{How to cite this article:}

Rashmi Padmanabha Mahale, Morubagal Raghavendra Rao and Sowmya Govindanahalli Shivappa. 2018. Evaluation of Procalcitonin, CRP and Blood Culture in the Diagnosis of Neonatal Sepsis. Int.J.Curr.Microbiol.App.Sci. 7(04): 2747-2755.

doi: https://doi.org/10.20546/ijcmas.2018.704.313 